\title{
Momentum-Resolved STM Studies of Rashba-Split Surface States on the Topological Semimetal Sb
}

\author{
Anjan Soumyanarayanan ${ }^{1,2, *}$ and Jennifer E. Hoffman ${ }^{1, \dagger}$ \\ ${ }^{1}$ Department of Physics, Harvard University, Cambridge, MA 02138, USA \\ ${ }^{2}$ Data Storage Institute, 5 Engineering Drive 1, Singapore 117608
}

\begin{abstract}
Topological materials host protected surface states with locked spin and momentum degrees of freedom. The helical Dirac character of the surface states, of tremendous scientific interest, stems from the interplay of the bulk band structure and surface Rashba spin-orbit interaction. The semimetal $\mathrm{Sb}$ offers a pristine platform to examine the Rashba origins of the Dirac-like topological surface states. Here we present an overview of our momentum-resolved scanning tunneling spectroscopy studies of $\mathrm{Sb}$, over an extended $(300 \mathrm{meV})$ energy range, revealing several features characteristic of the emergence of the Dirac-like surface states from a conventional Rashba-type parabolic dispersion. Our work provides a conceptual framework to create and investigate tunable Rashba states with topological properties.
\end{abstract}

The advent of a new topological class of electronic materials has created much excitement[1-4]. Strong spin-orbit coupling inverts the bulk valence and conduction bands in these materials, and their topological character necessitates the existence of protected surface states spanning the bulk band gap[5]. These topological surface states are spin-polarized, and typically have a near-linear energy-momentum relationship (Dirac dispersion) with their crossing at the Dirac point protected by time-reversal symmetry[1, 2, 5]. The exotic properties stemming from these topological materials have provided avenues to create and detect novel physical phenomena, and potential applications such as dissipationless spintronics $[1,2]$.
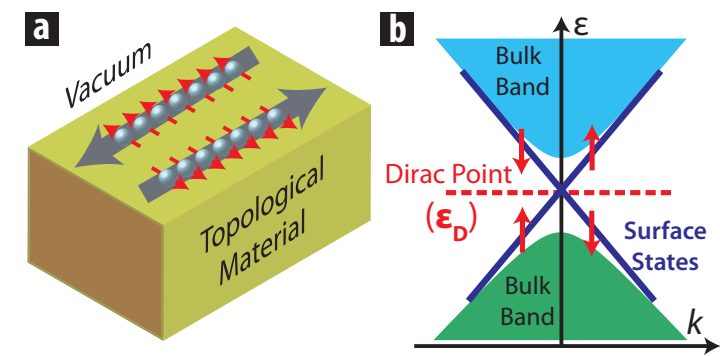

Figure 1. Schematic Representation of Topological surface states. (a) A cartoon illustration of a topological material, with protected, counterpropagating spin-polarized states at the surface. (b) Schematic band structure of a prototypical topological insulator (TI), with surface states (dark blue) spanning the bulk band gap and intersecting at the Dirac point, $\varepsilon_{\mathrm{D}}$.

\section{A. RASHBA EFFECT \& SURFACE STATE BAND STRUCTURE}

Surface states in topological materials exist as 'edge states' at the boundary of a bulk system[5], in contrast to the intrinsically 2D helical Dirac states in atomically thin graphene. Therefore, while the Dirac disper- sion in graphene arises from a simple tight-binding lattice model, it appears in topological materials due to surface-related symmetry considerations[6]. In particular, inversion symmetry is broken at the surface, and the resulting electric field couples directly to the spin of the relativistically moving electrons, a phenomenon known as the Rashba spin-orbit interaction[7]. The time-reversal invariant Dirac Hamiltonian thus obtained, $\mathcal{H}_{\mathrm{D}} \propto v_{0} \cdot(\vec{k} \times \vec{\sigma})$, where $v_{0}$ is the Rashba parameter, represents the locking of spin and momentum degrees of freedom, and results in near-linear, spin-split surface state dispersion[7, 8]. In fact, it is well known that even in conventional materials e.g. $\mathrm{Au}(111)$ and $\mathrm{Bi}(111)$, Rashba spin-orbit coupling leads to spin-polarized surface states[9]. The distinguishing characteristic of topological surface states is that are required to span the band gap, connecting the bulk valence and conduction bands $[1-3,6]$. This interplay of surface and bulk band structures leads to diverse manifestations of topological surface states across materials $[4,10]$.

\section{$k \cdot p$ Description of Surface States}

The simplistic Dirac Hamiltonian $\left(\mathcal{H}_{\mathrm{D}}\right)$ is inadequate for describing the rich phenomenology of topological surface states. For example, the momentum $(k-)$ space contours of constant energy, while circular near the Dirac point, are warped further from it $[11,12]$. Moreover, the shape and curvature of the surface state 'Dirac' cones vary substantially across different topological materials $[4$, 10]. These observations have been described using a phenomenological $k \cdot p$ Hamiltonian, which can be constructed by writing down all terms up to third order that are consistent with the time-reversal symmetry of the topological surface state, and the $\mathbb{C}_{3}$ crystal symmetry[8]

$$
H(k)=\varepsilon_{\mathrm{D}}+\frac{k^{2}}{2 m^{*}}+v_{0}\left(1+\alpha k^{2}\right)\left(k_{x} \sigma_{y}-k_{y} \sigma_{x}\right)+\frac{\lambda}{2}\left(k_{+}^{3}+k_{-}^{3}\right) \sigma_{z}
$$




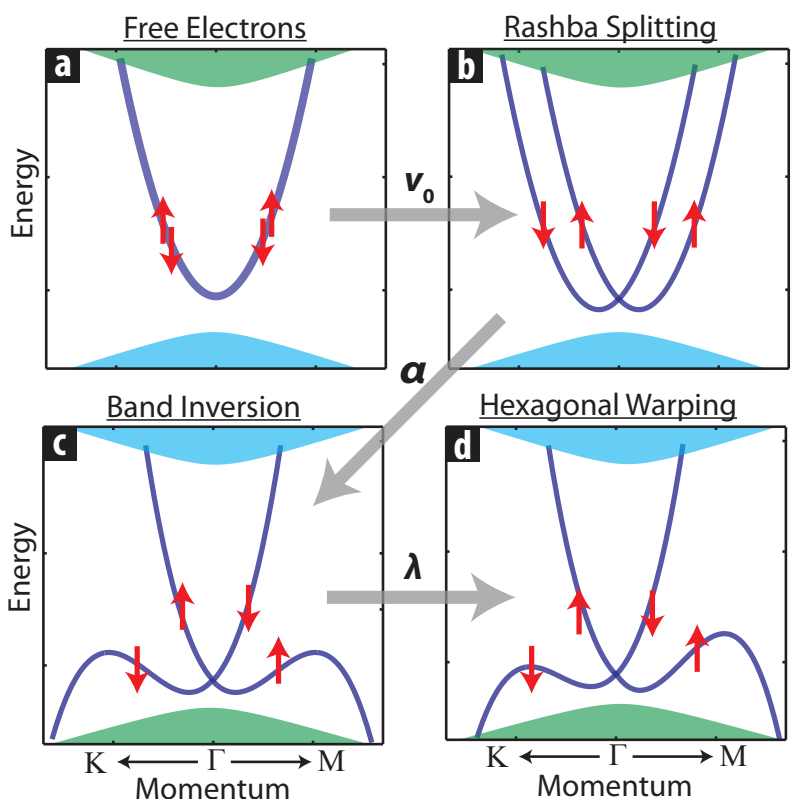

Figure 2. k.p Model of Topological Surface States. Schematic description of the emergence of topological surface states from free electrons using the Fu $k \cdot p$ model (Eqn. 1,[8]). The parabolic dispersion (a) is split by Rashba spin-orbit coupling (b). The outer Rashba cone is folded over by a cubic velocity correction (c), and hexagonal warping introduces anisotropy between $\Gamma-\mathrm{K}$ and $\Gamma-\mathrm{M}$ directions (d).

The terms consist of the linear, isotropic Rashba Hamiltonian, $\mathcal{H}_{\mathrm{D}} \propto v_{0}\left(k_{x} \sigma_{y^{-}} k_{y} \sigma_{x}\right)$ a correction of the Dirac velocity, $v(k)=v_{0}\left(1+\alpha k^{2}\right)$; a mass term, $\mathcal{H}_{m}=k^{2} / 2 m^{*}$ that creates particle-hole anisotropy about the Dirac point; and a warping term, $\mathcal{H}_{\mathrm{w}}=\lambda\left(k_{+}^{3}+k_{-}^{3}\right) \sigma_{z}$, which breaks rotational symmetry. This introduces the following five material parameters: the Dirac point energy, $\varepsilon_{\mathrm{D}}$; the effective mass, $m^{*}$; the Rashba parameter, $v_{0}$; the velocity correction, $\alpha$; and the warping strength, $\lambda[8]$.

It is instructive to visualize the emergence of the topological surface state bands from 2D free electrons, as shown in Fig. 2. Commencing with a parabolic spin-degenerate dispersion (Fig. 2a), we first include the Rashba parameter $\left(v_{0}\right)$ due to broken inversion symmetry, to obtain spin-split surface state parabolas (Fig. 2b)[7]. Next, the requirement that topological surface states connect the valence and conduction bands is satisfied by the $k^{3}$ velocity correction parameter $(\alpha)$, which folds down the outer Rashba cone (Fig. 2c). Finally, the addition of the warping parameter $(\lambda)$ introduces the observed $x-y$ anisotropy in surface state dispersion (Fig. 2d). Thus, while $m^{*}, \alpha$, and $\lambda$ control the shapes of the upper and lower Dirac cones, $v_{0}$ determines the splitting of the parent Rashba parabolas. This phenomenological $k \cdot p$ model is sufficiently general to describe and compare the surface states of various topological materials.

The Dirac fermion character of the topological surface states has been well-studied across several materials[11, 13-15]. However, the development of topological materials has been driven primarily with the objective of making the surface states and Dirac point physics more accessible to transport measurements and applications[4]. Despite the advent of large band gap topological insulators (TIs), transport measurements of these materials have been plagued with several difficulties. Most notably, the presence of intrinsic growth defects pins the chemical potential to the bulk conduction band, considerably diminishing the relative contribution of surface states to transport[16]. The intial use of chemical doping to tune the chemical potential into the bulk gap undesirably reduces the mean free path in several TIs[17]. Even the subsequent development of stoichiometric ternary and quaternary TIs $[18,19]$ has limited utility in circumventing this issue, as transport techniques remain sensitive to sample preparation[14, 20, 21]. Recent efforts to realize robust topological surface states have turned to developing materials with much larger Rashba parameters[22$24]$ and stack engineering of strong spin-orbit coupling materials[25-27]. It is therefore of tremendous value to understand the behavior of topological surface states in the context of their Rashba origin. Such an investigation would benefit from access to pristine surface states, unaffected by extrinsic artifacts such as chemical potential variations.

\section{From Rashba to Dirac Surface States}

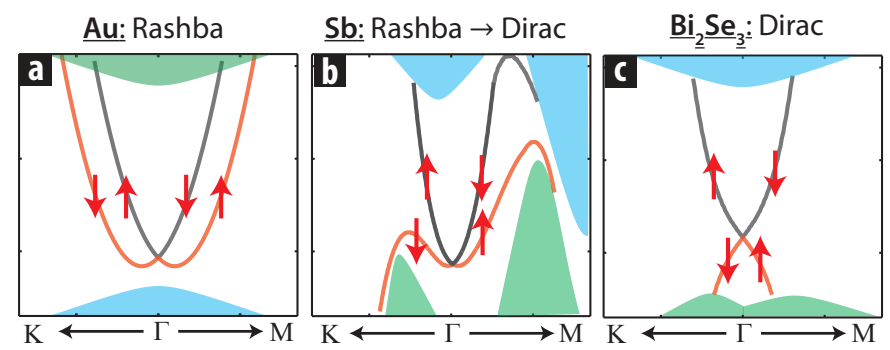

Figure 3. Connecting Rashba \& Dirac States. Schematics of the surface state band structures for (a) $\mathrm{Au}(111)$ (conventional Rashba system); (b) $\mathrm{Sb}(111)$; and (c) $\mathrm{Bi}_{2} \mathrm{Se}_{3}$ (near Dirac dispersing system). Sb provides a conceptual link between conventional Rashba surface states (near Dirac point) and Dirac dispersing topological surface states (well above Dirac point).

Antimony (Sb) offers an ideal platform for conceptualizing the emergence of topological surface states from the Rashba picture. Unlike TIs e.g. $\mathrm{Bi}_{2} \mathrm{X}_{3}$ and $\mathrm{Bi}_{x} \mathrm{Sb}_{1-x}$ $(x<0.2)$, which are semiconductors with a bulk band gap, $\mathrm{Sb}$ is a semimetal with a negative band gap, i.e. the valence and conduction bands overlap in energy (Fig. 3b, [28]). However, like the TIs, the bulk band structure of $\mathrm{Sb}$ is inverted by strong spin-orbit coupling and is topologically non-trivial. This peculiar topological, yet negative gap bulk band structure in Sb causes the surface 
states, which are required to connect the valence and conduction bands, to deviate markedly from the ideal Dirac cone structure found in existing TIs (Fig. 3b, c.f. Fig. 3c). In fact, the spin-split parabola band structure near the Dirac point is reminiscent of conventional Rashba systems (e.g. Fig. 3a). Meanwhile, its topological nature requires a single Dirac cone to persist at high energies, as in the case of TIs (e.g. Fig. 3c).

With characteristics of both Rashba and Dirac systems which evolve as a function of energy, Sb provides the opportunity to conceptually connect these frameworks. However, visualizing this dual behavior, which develops over several hundred meV about the Fermi energy $\varepsilon_{\mathrm{F}}$, requires a surface-sensitive spectroscopic tool with momentum-resolved access to both filled and empty states.

\section{B. SPECTROSCOPIC STM \& MOMENTUM RESOLUTION}

The scanning tunneling microscope (STM) has been used extensively over the past three decades as an atomic scale structural and electronic probe[29]. When an atomically sharp STM tip is held a distance $z$ (a few angstroms) from an atomically smooth sample surface at bias $V$, the tip-sample tunneling current $I(z, V)$ is given by $[30]$ :

$$
I(z, V) \propto \exp (-2 \kappa z) \int_{0}^{e V} d \varepsilon D_{\mathrm{s}}(\varepsilon)
$$

The exponential term $\exp (-2 \kappa z), \kappa$ being the local tunnel barrier height, provides picometer sensitivity to sample corrugations, facilitating atomically resolved structural imaging. Meanwhile, the integral term enables the use of STM as an nanoscale spectroscopic probe, measuring the single particle density of states, $D_{\mathrm{s}}(\varepsilon)$. Notably, STM can also provide $k$-space spectroscopic information through quantitative measurements of two phenomena.

\section{Landau Level Quantization}

First, the application of a sufficiently large magnetic field $B$, quantizes the density of states into Landau levels (LLs), which manifest as oscillations in the STM conductance $(d I / d V)$ spectra[31]. LLs correspond to quantized orbits in real and $k$-space[31]. In the semiclassical limit (large $n$ ), the Bohr-Sommerfeld quantization relation gives the area in $k$-space for the $n^{\text {th }} \mathrm{LL}[32]$ :

$$
A_{n} \equiv \pi q_{n}^{2}=(n+\gamma) \cdot 2 \pi e B / \hbar
$$

The factor $\gamma$, determined by the Berry's phase of the quasiparticle, is $1 / 2$ for a free electron and 0 for a Dirac fermion, empirically valid even for small $n[32,33]$. Thus, the LL dispersion can be used to extract the momentumresolved band structure of closed contours[32-34].

\section{Quasiparticle Interference}

Disorder can limit the formation of extended LLs; however it can also increase the prominence of a second $k$ space technique - quasiparticle interference (QPI) imaging. When quasiparticle states of energy $\varepsilon$ scatter elastically from impurities, the interference between initial and final quasiparticle wavevectors $\vec{k}_{\mathrm{i}}$ and $\vec{k}_{\mathrm{f}}$ can result in a standing wave pattern with wavevector $\vec{q}=\vec{k}_{\mathrm{f}}-\vec{k}_{\mathrm{i}}$ at energy $\varepsilon$. This can be visualized in an STM $d I / d V$ map at energy $\varepsilon=e V[35]$. The observed dispersion of $q(\varepsilon)$ with energy, and the inversion of $q(\varepsilon)$ to find $k(\varepsilon)$ has established QPI as a reliable $k$-space probe of electronic materials[36-38].

The nanoscale spatial resolution, temperature-limited energy resolution, access to filled and empty states, and utility in magnetic field offered by STM measurements of LLs and QPI make them ideal complements to angleresolved photoemission spectroscopy as band structure probes. In fact, LL spectroscopy and QPI imaging have shed light on several aspects of Dirac materials. For example, they have been used to visualize the absence and onset of backscattering in topological materials[39-41], pseudospin protection in graphene[38, 42] and chemical potential fluctuations in a range of materials[17, 42, 43]. While each of these techniques offers great promise on its own for measuring simpler band structures, the synergistic utilization of LLs and QPI could also enable the determination of multi-component band structures. This is especially useful to investigate the varied manifestations and tunability of Rashba-split topological surface states.

Here, we describe our momentum-resolved STM investigation of the surface states of the topological semimetal $\mathrm{Sb}[44]$. Our observations of simultaneous Landau quantization and QPI over an extended (300 meV) energy range reveal several idiosyncrasies of the topological surface states of $\mathrm{Sb}$, arising from their dual (Rashba $\rightarrow$ Dirac) nature. We further employ the complementary $k$-space sensitivity of these momentum-resolved phenomena to detail the surface state band structure, relating it to the $k \cdot p$ description.

\section{TOPOGRAPHY \& SPECTROSCOPIC CHARACTERIZATION}

\section{STM Topography}

The (111) surface of Sb shows large atomically flat regions(Fig. 4a, [45]) forming a hexagonal lattice with the 


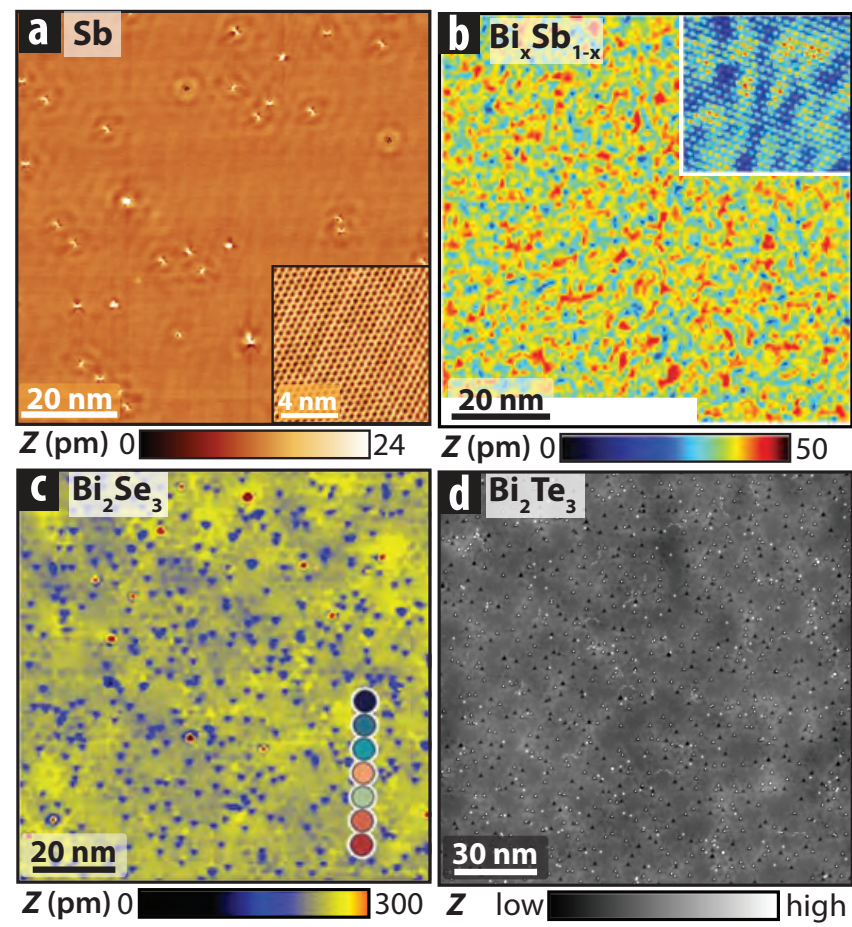

Figure 4. Topography: Sb and other Topological Materials. (a) STM topograph of Sb showing an atomically flat surface. Inset shows the atomically resolved hexagonal (111) lattice. (b-d) STM topographs acquired on other topological materials: (b) $\mathrm{Bi}_{x} \mathrm{Sb}_{1-x}$ [39]; (c) doped $\mathrm{Bi}_{2} \mathrm{Se}_{3}$ [17]; and (d) doped $\mathrm{Bi}_{2} \mathrm{Te}_{3}$ [41]. All three topographs show aperiodic modulations attributed to chemical potential fluctuations[17].

expected periodicity $\left(a_{0}=4.31 \AA\right)$. Over flat regions of the sample, topographic modulations correspond directly to visible single-atom impurities (Fig. 4a), which do not noticeably change the local chemical potential[44].

A comparison of the corrugations observed in STM topographs of Sb with those reported on TIs (Fig. 4b-d) is rather striking. All these materials have approximately similar elemental compositions and lattice dimensions; the structural features and corrugations are expected to be similar. However, the electronic contribution to STM topographs in TIs, which typically manifests as aperiodic inhomegenous patches, is notable in $\mathrm{Bi}_{2} \mathrm{X}_{3}[17,41]$ and provides the dominant contribution to the topographs in $\mathrm{Bi}_{x} \mathrm{Sb}_{1-x}[39]$. Such electronic inhomogeneity in TIs is understood to be associated with poorly screened bulk defects[17]. The contrasting topographs imaged on $\mathrm{Sb}$ with much reduced spatial variations suggest that the surface chemical potential here is homogeneous in comparison to TIs. This homogeneity is associated with the presence of bulk bands proximate to the surface states, which provide screening.

\section{STM Spectroscopy}

A typical $d I / d V$ spectrum acquired on $\mathrm{Sb}$ exhibits prominent cusp-like features, as shown in Fig. 5a. We
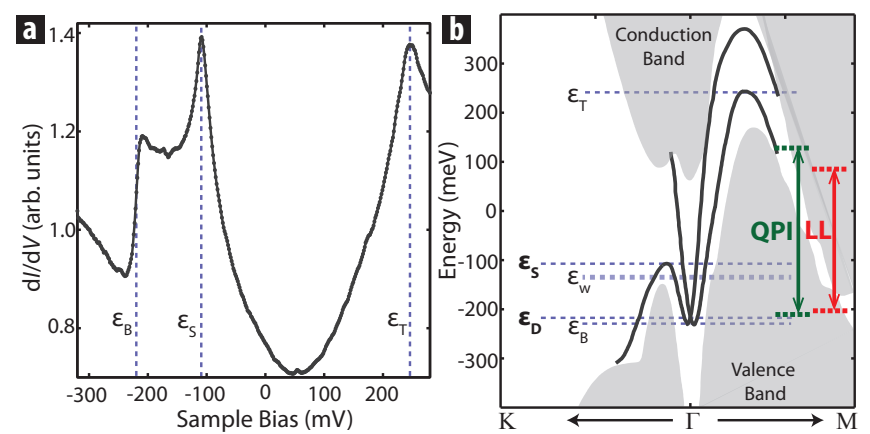

Figure 5. Sb Spectroscopy and Band Structure. (a) A typical $d I / d V$ spectrum, with cusp-like features at $\varepsilon_{\mathrm{B}}, \varepsilon_{\mathrm{S}}$, and $\varepsilon_{\mathrm{T}}$, corresponding to extremal surface state features in (b). (b) Schematic band structure of $\mathrm{Sb}(111)$ with topological surface states (dark grey) intersecting at the Dirac point, $\varepsilon_{\mathrm{D}}$.

note that the measured $d I / d V$ could have contributions from both surface and bulk bands. To understand the origin of these features and the overall spectral shape, we turn to a schematic of the Sb surface and bulk band structure (Fig. 5b), obtained from first principles density functional theory slab calculations[44].

The bulk semimetallic character of $\mathrm{Sb}$ is evident in Fig. $5 \mathrm{~b}$ from the $>300 \mathrm{meV}$ overlap between the valence and conduction bands. As detailed in Sec. A, the negative bulk band gap distorts the topological surface states spanning the gap, producing a pair of Rashba-split cones near the Dirac point. The inner cone is allowed to persist up to much higher energies, while the outer cone is required to fold down in order to connect to the valence band. This results in the formation of extremal features in the outer Rashba cone, one of which is a saddle point $\left(\varepsilon_{\mathrm{S}}\right)$, and the other is a band edge $\left(\varepsilon_{\mathrm{T}}\right)$. The energy difference between these two surface state extrema $\varepsilon_{\mathrm{S}}$ and $\varepsilon_{\mathrm{T}}$ is due to the strength of the warping term (Eqn. 1).

Therefore, the cusp-like features observed in $d I / d V$ spectra (Fig. 5a) can be well-explained by the extrema in the surface state band structure (Fig. $5 \mathrm{~b}$ ). The $d I / d V$ steps at $-220 \mathrm{mV}$ and $240 \mathrm{mV}$ are associated with $2 \mathrm{D}$ band edges at $\varepsilon_{\mathrm{B}}$ and $\varepsilon_{\mathrm{T}}$ respectively, while the $d I / d V$ peak at $-110 \mathrm{mV}$ corresponds to the logarithmic singularity associated with the $2 \mathrm{D}$ saddle point at $\varepsilon_{\mathrm{S}}$. We note in particular the importance of this saddle point, which signifies a crossover between the appearance of the surface state bands as Rashba-split parabolas (below $\varepsilon_{\mathrm{S}}$ ) to an isolated Dirac cone (above $\varepsilon_{\mathrm{S}}$ ).

The comparison of the Sb spectrum with TIs brings forth several illuminating facts. First, we observe a much larger $d I / d V$ background in Sb relative to TIs, which likely comes from proximate bulk bands. Next, we note that all prominent features in $\mathrm{Sb}$ over a $400 \mathrm{meV}$ energy range can be ascribed to surface state extrema. In contrast, bulk band extrema typically produce noticeable spectral kinks in TIs. Finally, we note the absence 

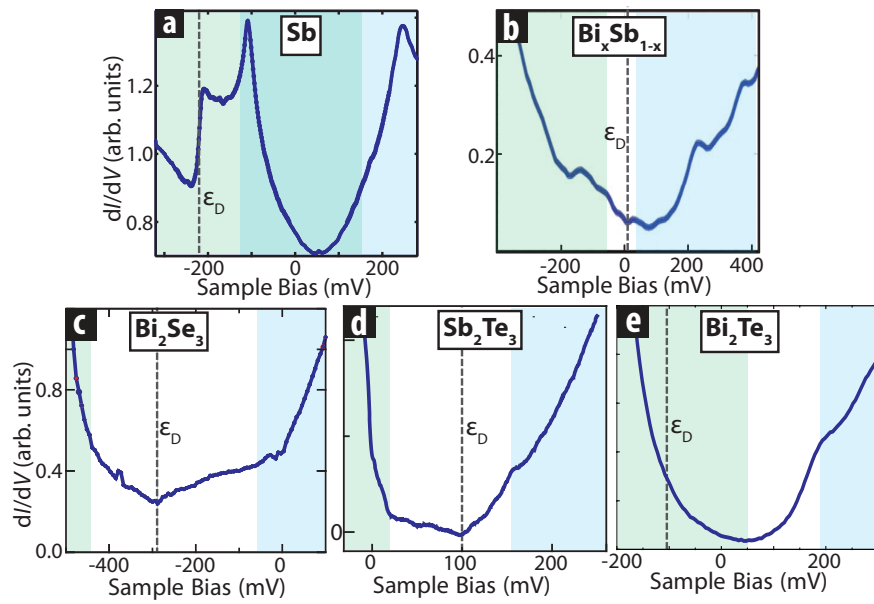

Figure 6. STM Spectra across Topological Materials. STM $d I / d V$ spectrum on (a) Sb [44] compared to TIs: (b) $\mathrm{Bi}_{x} \mathrm{Sb}_{1-x}$ [39]; (c) $\mathrm{Bi}_{2} \mathrm{Se}_{3}$ [32]; (d) $\mathrm{Sb}_{2} \mathrm{Te}_{3}[15]$; and $\mathrm{Bi}_{2} \mathrm{Te}_{3}[41]$. The dashed vertical line indicates the Dirac point, $\varepsilon_{\mathrm{D}}$, which corresponds to the spectral minimum in (cd). Shaded green and blue regions represent valence and conduction bands respectively. Note that in (b-e), bulk band extrema are typically associated with spectral kinks.

of a $d I / d V$ minimum associated with the Dirac point in $\mathrm{Sb}$, due its spectral coincidence with other surface states. This is in marked contrast to the STM spectra acquired on TIs $\mathrm{Bi}_{2} \mathrm{Se}_{3}$ and $\mathrm{Sb}_{2} \mathrm{Te}_{3}[15,32]$ : Fig. 6c-d), where the bulk band gap and conventional Dirac cone structure result in a $d I / d V$ minimum at $\varepsilon_{\mathrm{D}}$. While the bulk continuum and Rashba form of Sb surface states at lower energies do limit direct Dirac point access for $d I / d V$ measurements, we will show that they provide distinct advantages for momentum-resolved measurements by screening out tip-induced band bending artifacts[46] and chemical potential fluctuations[17, 39], and providing quasiparticle scattering channels to probe near-Dirac point physics.

\section{LANDAU LEVEL QUANTIZATION SPECTROSCOPY}

\section{Landau Level Quantization Results}

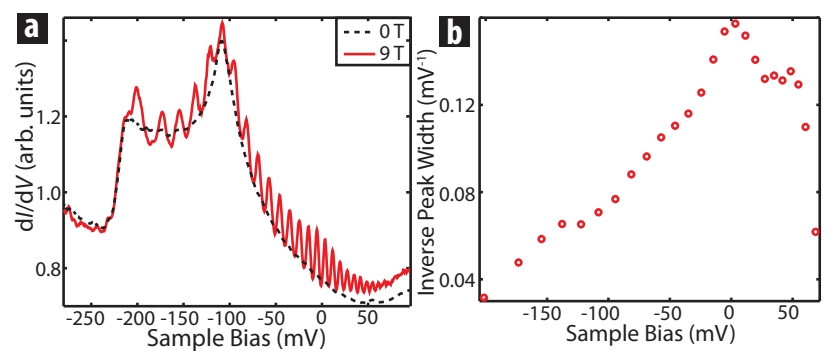

Figure 7. Landau Quantization in Sb. (a) Typical $d I / d V$ spectrum acquired at $9 \mathrm{~T}$ (red) (c.f. $0 \mathrm{~T}$, dashed black), showing LL oscillations. (b) LL inverse peak widths, obtained by fitting the data in (a), showing lifetime broadening away from $\varepsilon_{\mathrm{F}}$.
In the presence of sufficiently large perpendicular magnetic fields, oscillations corresponding to quantized Landau levels (LLs) are observed in $d I / d V$ spectra on $\mathrm{Sb}$ (Fig. 7a). The LLs emerge as modulations on top of the background (zero field) spectrum, and are unequally spaced in energy, as expected for Dirac fermion surface states. We observe up to 27 such LLs - more than the number reported in any TI (Tbl. I). It is worth noting that the record number of LLs seen in $\mathrm{Sb}$ is despite the presence of bulk bands throughout this energy range (Fig. 5b). In comparison, LLs in TIs are typically observed over the spectral range corresponding to the bulk band gap $[15,32]$.

\begin{tabular}{|c|c|c|c|}
\hline Material & \# of LLs & Field (T) & Ref. \\
\hline \hline $\mathrm{Bi}_{2} \mathrm{Se}_{3}$ & 23 & 11 & {$[32,46]$} \\
\hline $\mathrm{Bi}_{2} \mathrm{Te}_{3}$ & 13 & 7 & {$[34]$} \\
\hline $\mathrm{Sb}_{2} \mathrm{Te}_{3}$ & 15 & $6-7$ & {$[15]$} \\
\hline $\mathrm{Bi}_{2} \mathrm{Te}_{2} \mathrm{Se}$ & 18 & 11 & {$[47]$} \\
\hline $\mathbf{S b}$ & $\mathbf{2 7}$ & $\mathbf{6 - 9}$ & {$[\mathbf{4 4}]$} \\
\hline
\end{tabular}

Table I. A comparison of the number of LLs reported in various spin-orbit coupled topological materials.

The LL positions and widths are extracted by fitting normalized spectra to a sum of Lorentzian profiles (details in [44]). The LL peaks are found to be sharpest at the Fermi energy, $\varepsilon_{F}$. The inverse LL peak width, a measure of the quasiparticle lifetime, is peaked at $\varepsilon_{\mathrm{F}}$ and broadens away from it (Fig. 7b). The lifetime broadening is monotonic away from $\varepsilon_{\mathrm{F}}$, in contrast to TIs where collective modes complicate the picture[32, 34]. This can be well explained within the Fermi liquid paradigm, where quasiparticles with energy $\varepsilon$ relative to $\varepsilon_{\mathrm{F}}$ decay by creating particle-hole excitations. The phase space of available excitations results in the quasiparticle lifetime having a $1 / \varepsilon^{2}$ energy dependence, which adequately describes these observations. Finally, the measured lifetime at $\varepsilon_{\mathrm{F}}\left(\sim 0.16 \mathrm{mV}^{-1}\right)$ corresponds to a long elastic free path, $\ell_{\mathrm{f}} \sim 65 \mathrm{~nm}$.

\section{LL Dispersion: Dirac Regime}

As noted previously, LLs correspond to closed contours in $k$-space, and can be used to obtain the dispersion of topological surface states. The dispersion of LLs in several TIs has been interpreted within the isotropic Dirac fermion picture[15,32, 41, 46], with the energy of the $n^{\text {th }}$ $\mathrm{LL}, \varepsilon_{n}$, given by

$$
\varepsilon_{n}(B)=\varepsilon_{\mathrm{D}}+v_{\mathrm{D}} \sqrt{2 e \hbar n B}
$$

where the Fermi velocity $v_{\mathrm{D}}$ is a constant over the energy range of interest. Meanwhile, the Bohr-Sommerfeld quantization relation for Dirac fermions gives the $k$-space radius for the $n^{\text {th }}$ LL orbit to be: $q_{n}=\sqrt{(2 e / \hbar) n B}[32]$. This enables the determination of $\varepsilon_{n}\left(q_{n}\right)$ - the quasipar- 

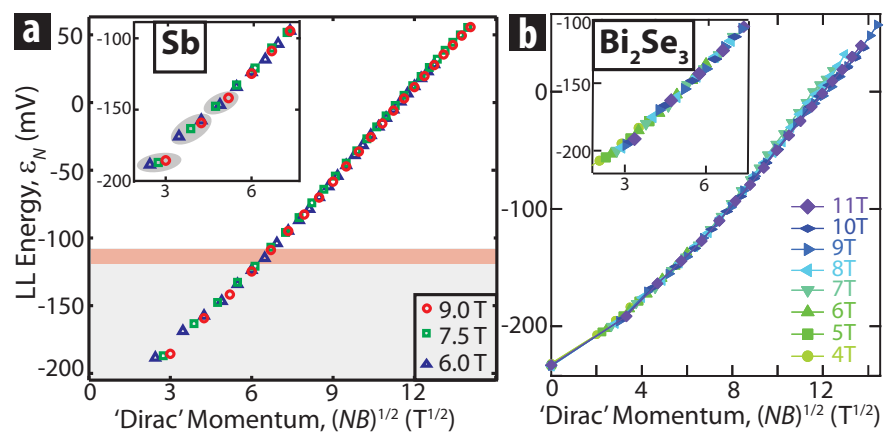

Figure 8. LL Dispersion Comparison. (a) Dispersion of LL energies $\varepsilon_{N}$ vs. Dirac fermion momentum $\sqrt{N B}$ (Eqn. 4) for $\mathrm{Sb}[44]$. Light orange band shows the crossover energy for the Rashba regime (Eqn. 5). (b) Analogous LL dispersion plot for $\mathrm{Bi}_{2} \mathrm{Se}_{3}$ [32], which shows Dirac dispersion throughout its energy range. Insets (a-b) focus on the LL dispersion near the Dirac point.

ticle band structure - so long as $q_{n} \sim \sqrt{n B}$ is a good quantum number[48].

Fig. 7a shows the LL peak energies, $\varepsilon_{N}$, plotted against the empirical LL momentum, $q_{N}=\sqrt{N B}$, where $N$ is the empirical LL index, counted from the lowest observed LL. For energies $\varepsilon>\varepsilon_{\mathrm{S}}$, the dispersions obtained at different magnetic fields collapse on to a single curve - validating the Dirac fermion approximation with $n=N$ (fit details in [44]). The correspondence of the LL dispersion of $\mathrm{Sb}$ with prototypical TIs (Fig. 8b, [32]) for $\varepsilon>\varepsilon_{\mathrm{S}}$ demonstrates that the Landau quantization arises from a single Dirac cone in this energy range. We conclude that the LL wavevector $q_{N}$ corresponds to the radius of the inner cone, and gives its velocity, $v_{\mathrm{LL}} \equiv v_{\mathrm{D}}=4.20 \mathrm{eV} \cdot \AA$ $\left(6.34 \times 10^{5} \mathrm{~m} / \mathrm{s}\right)$.

\section{LL Dispersion: Rashba Regime}

For energies $\varepsilon<\varepsilon_{\mathrm{S}}$ however, the dispersion systematically deviates from the $\sqrt{N B}$ fit, with much reduced field dependence upon approaching the Dirac point (Fig. 8a, inset). This is in stark contrast to the consistent $\sqrt{N B}$ dispersion observed in the single Dirac cone picture (e.g. Fig. 8b, inset). Having ruled out the presence of extrinsic artifacts, such as chemical potential fluctuations, that may complicate the interpretation of LLs in TIs, e.g. $\mathrm{Bi}_{2} \mathrm{Te}_{3}[34]$, we conclude that the Dirac momentum $\sqrt{N B}$ is not a valid descriptor for $\varepsilon<\varepsilon_{\mathrm{S}}$ in $\mathrm{Sb}$.

We therefore analyze the $\varepsilon<\varepsilon_{\mathrm{S}}$ LLs within the Rashba picture of spin-split parabolic bands[7]. The LLs in the Rashba picture derive from free fermions - i.e. the LLs, indexed by $n$, are first separated by the cyclotron energy, $\hbar \omega_{\mathrm{c}}$, with $\omega_{\mathrm{c}}=e B / m^{*}$. An additional term, dependent on the Rashba parameter $v_{0}$, further splits each LL $(n>$ 0 ) according to[49-51]:
$\varepsilon_{n}(B)= \begin{cases}\varepsilon_{0}+\frac{1}{2}\left(\hbar \omega_{\mathrm{c}}+g \mu_{\mathrm{B}} B\right), & n=0 \\ \varepsilon_{0}+\hbar \omega_{\mathrm{c}} n \pm \sqrt{\delta^{2} / 4+\left(2 m^{*} v_{0}^{2}\right) \cdot n \hbar \omega_{\mathrm{c}}}, & n>0\end{cases}$

Here, $\varepsilon_{0}$ is the band offset, $\delta=\left(1-1 / 2 g m^{*}\right) \hbar \omega_{\mathrm{c}}$, and $g$ is the electron g-factor[49,50]. The upper and lower Rashba-split branches qualitatively correspond to the inner and outer Rashba 'cones', respectively. Our measured LLs correspond to the positive sign in Eqn. 5, which derives predominantly from the inner Rashba cone (Fig. 11c). Meanwhile, the larger momenta and tighter energy spacing of the LLs corresponding to the outer cone, and their resultant susceptibility to broadening effects, is likely responsible for their absence in our spectra. Our LL results thus represent a smooth crossover between the Rashba and Dirac pictures.

Finally, we note that a unified expression has been derived to account for the non-linear dispersion of topological surface states (e.g. Fig. 8b)[48]. However, such an analytic formulation lacks $k$-cubic terms (Eqn. 1) and is therefore unable to describe the LL dispersion resulting from the turnover of spectrally coincident Rashba cones - as is the case in Sb. Moreover, the complexity of such an expression obscures the intuitive understanding of the two limiting (Rashba and Dirac) cases, arising from the simple physical picture in Fig. 2, which quantitative describe nearly all the LL data. Furthermore, the separation into these limiting cases also reduces the number of free parameters, thereby providing more reliable fit parameters.

\section{E. QUASIPARTICLE INTERFERENCE SPECTROSCOPY}

As exemplified in Fig. 9a-b, impurity scattering creates striking interference patterns in $d I / d V$ maps on $\mathrm{Sb}$. In the Fourier space $d I / d V$ maps shown in Fig. 9e-f, we note the presence of peaks along both the $\Gamma-\mathrm{M}$ and $\Gamma-\mathrm{K}$ reciprocal directions. $d I / d V$ linecuts extracted along these high-symmetry directions (Fig. 9e-f) show QPI modes as intensity peaks that disperse approximately linearly with energy over $\sim 300 \mathrm{mV}$ from the Dirac point, the $\Gamma-\mathrm{M}$ dispersion having been reported previously [52-54]. The prominent modes seen in such data sets are labeled as $q_{\Gamma-\mathrm{M}, 1}, q_{\Gamma-\mathrm{M}, 2}$, and $q_{\Gamma-\mathrm{K}}$ respectively. We also observe similar scattering patterns emanating from atomic steps $[52,53,55]$, with more pronounced signatures of the $q_{\Gamma-\mathrm{M}, 2}$ mode than in atomically flat regions (details in [44]).

A comparison of the QPI phenomenology of $\mathrm{Sb}$ (Fig. 10a) with several TIs (Fig. 10b-d) brings forth several contrasting observations. The existence of three QPI modes as opposed to the sole dispersing mode observed in 

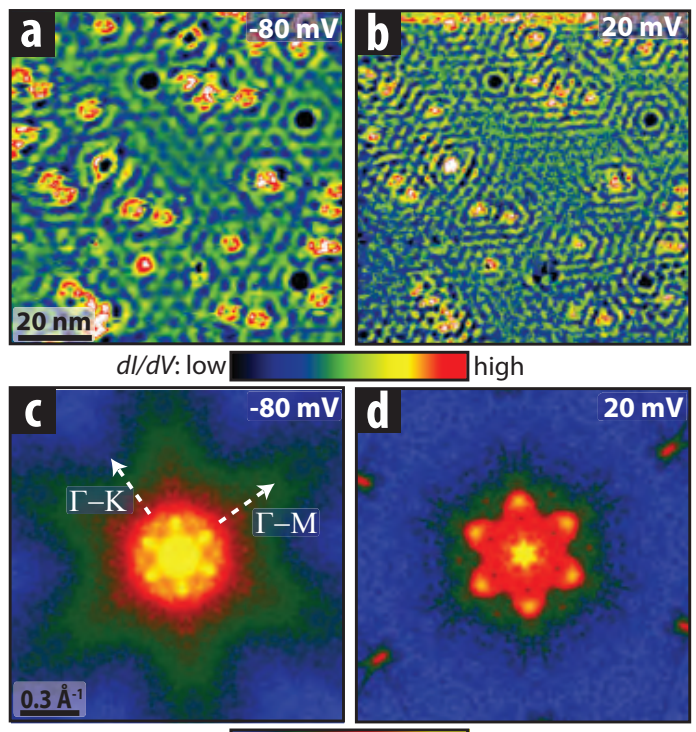

$d l / d V: \operatorname{low} \square$ high

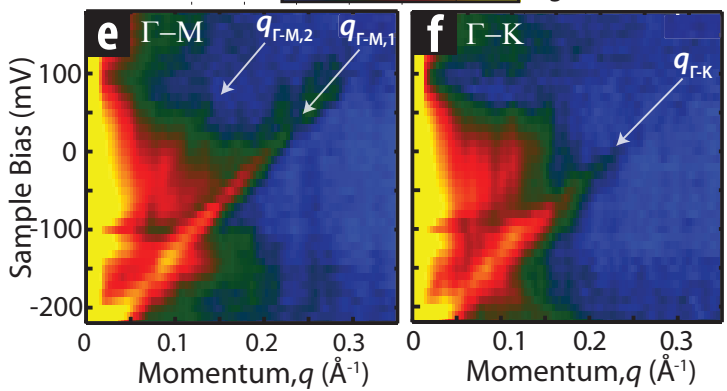

Figure 9. QPI of Sb Surface States. (a, b) $d I / d V(\vec{r}, V)$ maps at $V_{0}=-80 \mathrm{mV}$ (a) and $+20 \mathrm{mV}$ (b), showing standing wave patterns (QPI) around impurities. (c, d) Fourier Transforms (FTs) of (a) and (b), with distinct peaks along $\Gamma-\mathrm{M}$ and $\Gamma-\mathrm{K}$ directions. (e, f) $d I / d V$ linecuts through FTs along the $\Gamma-\mathrm{M}(\mathrm{e})$ and $\Gamma-\mathrm{K}$ (f), generated from $190 \mathrm{~nm}$ spatial maps. The prominent dispersing modes are indicated.

the $\mathrm{Bi}_{2} \mathrm{X}_{3}$ class (Fig. 10c-d) owes to the multi-component surface state band structure of Sb. Moreover, the QPI in Sb persists down to the $q \rightarrow 0$ limit - near the Dirac point - unlike in other TIs where QPI is limited to the range $q \gtrsim 0.1 \AA^{-1}$ and $\left(\varepsilon-\varepsilon_{\mathrm{D}}\right)>100 \mathrm{meV}$. There are two reasons for this contrast in the momentum and spectral extent of QPI. The momentum limitation in TIs arises from chemical potential fluctuations due to poor screening of bulk defects substantially reduce the mean free path, $\ell_{\mathrm{f}}$ in TIs. This imposes a lower bound for the scattering wavevector in TIs, $q_{\min } \sim \ell_{\mathrm{f}}^{-1}$, below which $q$ is no longer a good quantum number, and QPI cannot be observed. In contrast, the homogeneous surface of $\mathrm{Sb}$ results in a much lower scattering cut-off $\left(q_{\min } \sim 0.01 \AA^{-1}\right)$. Meanwhile, the energy limitation arises in TIs arises from the presence of an isolated, isotropic Dirac cone (i.e. a circular contour) in the $\mathrm{Bi}_{2} \mathrm{X}_{3}$ class. Such a contour does not have a coherent scattering profile that could produce static QPI patterns, since the dominant $2 k_{\mathrm{F}}$ backscattering mode is forbidden[40]. QPI in TIs is thus observed
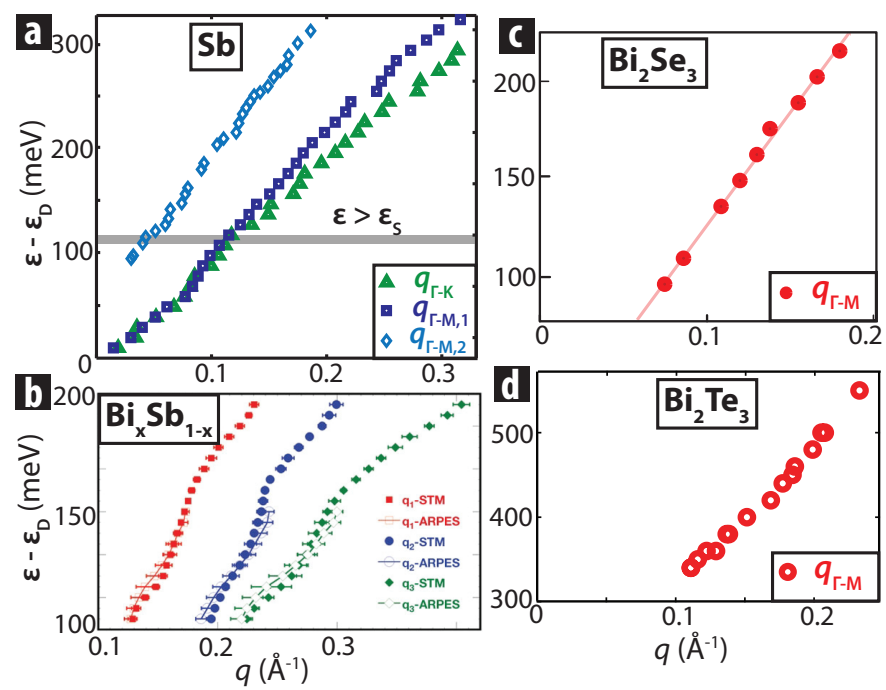

Figure 10. QPI Dispersion across Topological Materials. Compiled QPI modes observed on (a) Sb [44], compared to TIs: (b) $\mathrm{Bi}_{x} \mathrm{Sb}_{1-x}$ [39]; (c) $\mathrm{Bi}_{2} \mathrm{Se}_{3}$ [17]; and $\mathrm{Bi}_{2} \mathrm{Te}_{3}[41]$. Unlike $\mathrm{Sb}$, which has two near-Dirac point modes, QPI is not observed in TIs (b-d) for $q \gtrsim 0.1 \AA^{-1}$ and $\varepsilon-\varepsilon_{\mathrm{D}}>100 \mathrm{meV}$.

at much higher energies, where the constant energy contour deforms to produce scattering 'hot spots' $[8,12,40]$. However in $\mathrm{Sb}$, the presence of an outer Rashba cone for near-Dirac point energies allows for spin-conserved interband scattering (Fig. 11b, details in Sec. F), creating static QPI patterns. Thus, the outer Rashba cone in Sb allows the visualization of the Dirac point physics of topological surface states without breaking time-reversal symmetry.

\section{F. MOMENTUM-RESOLVED STM BAND STRUCTURE}

The $\varepsilon(q)$ dispersions of all quasiparticle modes $q_{\Gamma-\mathrm{M}, 1}, q_{\Gamma-\mathrm{M}, 2}, q_{\Gamma-\mathrm{K}}$, and $q_{\mathrm{LL}}-$ are shown in Fig. 11a. Notable features include (a) the onset of $q_{\Gamma-\mathrm{M}, 2}$ at a slightly higher energy, $\varepsilon_{\mathrm{w}}$, compared to the other QPI modes, and (b) the identical dispersion of $q_{\Gamma-\mathrm{M}, 1}(\varepsilon)$ and $q_{\Gamma-\mathrm{K}}(\varepsilon)$ for lower energies, and their deviation above $\varepsilon_{\mathrm{s}}$.

To identify the $k$-space origin of these dispersing modes, we examine schematics of the constant energy contours in Fig. 11b, which display three qualitatively different shapes over the energy range of interest. For $\varepsilon_{\mathrm{D}}<\varepsilon<\varepsilon_{\mathrm{w}}$, the contours are concentric spin-polarized circles corresponding to the spin-split Rashba parabolas. Here, the only allowed scattering mode is inter-band scattering between parallel spins. Therefore $q_{\Gamma-\mathrm{M}, 1}(\varepsilon)$ and $q_{\Gamma-\mathrm{K}}(\varepsilon)$ both correspond to this inter-band mode, and are identical. For $\varepsilon_{\mathrm{w}}<\varepsilon<\varepsilon_{\mathrm{S}}$, the outer Rashba cone acquires a warped snowflake shape, which corresponds to the onset of intra-band scattering on the outer 

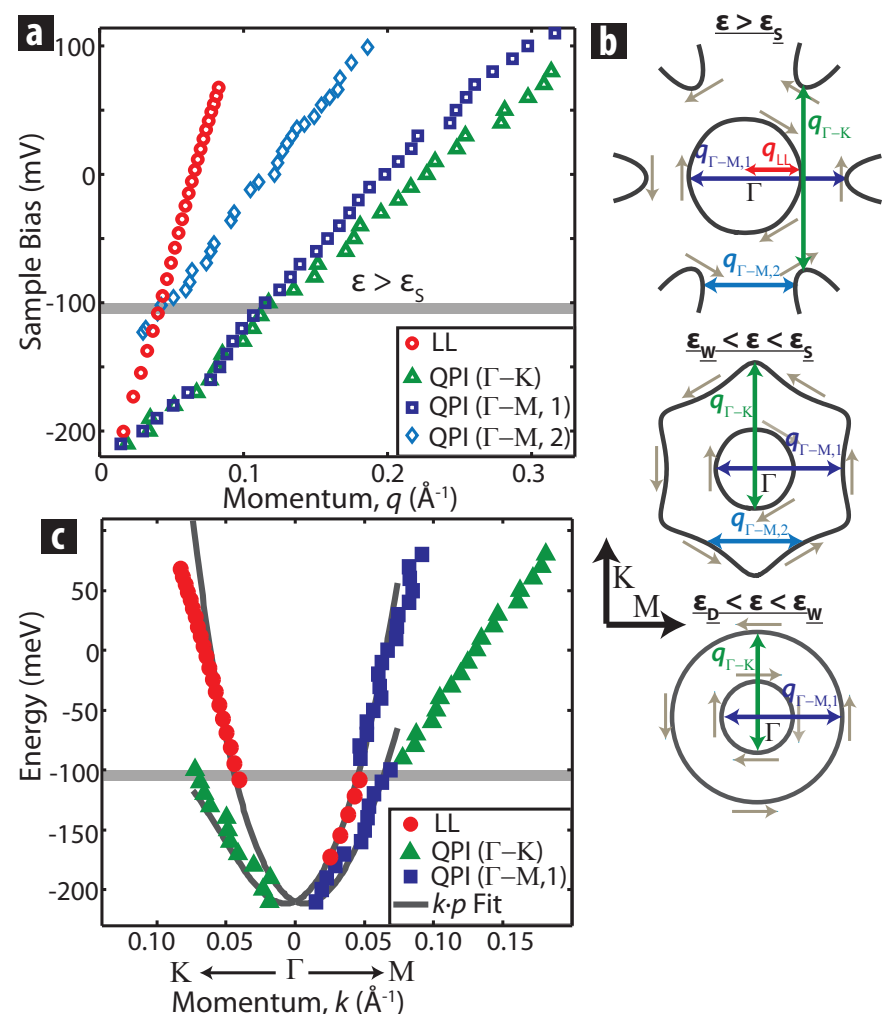

Figure 11. Sb LL and QPI Modes in $q$-space. (a) Compilation of dispersions recorded over the same spatial region using LLs and QPI. (b) Schematic evolution of the surface state contours of constant energy. The in-plane spin polarization is indicated in brown, with $q$-space locations of QPI and LL modes overlaid. (c) Momentum-resolved STM determination of the surface state band structure from Fig. 11a. Grey lines correspond to a $k \cdot p$ fit.

cone, observed as the $q_{\Gamma-\mathrm{M}, 2}(\varepsilon)$ mode[53]. Meanwhile, the $k$-space origin of $q_{\Gamma-\mathrm{M}, 1}(\varepsilon)$ and $q_{\Gamma-\mathrm{K}}(\varepsilon)$ is the same as before. Finally, for $\varepsilon>\varepsilon_{\mathrm{S}}$, the outer cone is no longer closed. While the $q_{\Gamma-\mathrm{M}, 1}(\varepsilon)$ mode remains the same as before, $q_{\Gamma-\mathrm{K}}(\varepsilon)$ now corresponds to intra-band scattering across the outer 'pocket', analogous to $q_{\Gamma-\mathrm{M}, 2}(\varepsilon)$. Above $\varepsilon_{\mathrm{S}}$, the LL wavevector, $q_{\mathrm{LL}}(\varepsilon)$, is well defined, and corresponds to the area of the inner pocket - the only closed contour over the energy range of interest.

Therefore, the dominant $\Gamma-\mathrm{M}$ QPI mode, $q_{\Gamma-\mathrm{M}, 1}(\varepsilon)$, corresponds to inter-band scattering between parallel spins over the entire observed energy range. In contrast, the $\Gamma-\mathrm{K}$ QPI mode $q_{\Gamma-\mathrm{K}}(\varepsilon)$ corresponds initially to inter-band scattering below $\varepsilon_{\mathrm{S}}$, and transitions to scattering between sections of the outer pocket for $\varepsilon>\varepsilon_{\mathrm{S}}$. We note the kink and slight change in magnitude of $q_{\Gamma-\mathrm{K}}(\varepsilon)$ around $\varepsilon_{\mathrm{S}}$ (Fig. 11), corresponding to this crossover from inter-pocket scattering to intrapocket scattering. This change in dispersion signifies the turnover of the outer Rashba cone, and represents the crossover to the Dirac regime of the topological surface state band structure. Such a crossover is in con- trast to QPI modes arising from prototypical Rashbasplit states in conventional materials[56], also known to exist on TIs $[20,21,57]$.

Having determined the origins of all observed $q$-vectors, we use $\vec{q}_{\mathrm{LL}}(\varepsilon), \vec{q}_{\Gamma-\mathrm{K}}(\varepsilon)$, and $\vec{q}_{\Gamma-\mathrm{M}, 1}(\varepsilon)$ in conjunction with geometric considerations to extract the three independent components of the $\varepsilon>\varepsilon_{\mathrm{S}}$ band structure. Next, we use the LL and QPI techniques in concert to determine the band structure in the Rashba $\left(\varepsilon<\varepsilon_{\mathrm{S}}\right)$ regime within the $k \cdot p$ formalism (Fig. 11c). Our method uniquely utilizes the complementary $k$-space origins of QPI and LLs to determine the various $k \cdot p$ parameters, employing (a) $\Gamma-\mathrm{M}$ and $\Gamma-\mathrm{K}$ QPI modes, which arise from intra-parabola scattering, to obtain the effective mass $\left(m^{*}=m / m_{\mathrm{e}}=0.1\right)$, warping parameter $\left(\lambda=200 \AA^{-3}\right)$, and the velocity correction $\left(\alpha=130 \AA^{-2}\right)$; and (b) the LL dispersion (Eqn. 5) directly resulting from the interparabola Rashba splitting, to find the Rashba parameter, $v_{0}=0.51 \mathrm{eV} \cdot \AA\left(7.7 \times 10^{4} \mathrm{~m} / \mathrm{s}\right)$ and Dirac point, $\varepsilon_{\mathrm{D}}=-210 \mathrm{meV}$. The correspondence between the $k \cdot p$ fit and the measured LL data is shown in Fig. 11c.

\begin{tabular}{|c|c|c|c|c|c|c|}
\hline Material & $\begin{array}{c}\varepsilon_{\mathrm{D}} \\
(\mathrm{meV})\end{array}$ & $\begin{array}{c}m^{*} \\
\left(m / m_{e}\right)\end{array}$ & $\begin{array}{c}v_{0} \\
(\mathrm{eV} \cdot \AA)\end{array}$ & $\begin{array}{c}\alpha \\
\left(\AA^{-2}\right)\end{array}$ & $\begin{array}{c}\lambda \\
\left(\mathrm{eV} \cdot \AA^{-3}\right)\end{array}$ & Ref. \\
\hline \hline $\mathrm{Bi}_{2} \mathrm{Se}_{3}$ & -300 & - & 0.47 & 743 & 125 & {$[58]$} \\
\hline $\mathrm{Bi}_{2} \mathrm{Te}_{3}$ & -105 & 0.5 & 0.32 & 210 & 137 & {$[34]$} \\
\hline $\mathrm{Sb}$ & -210 & 0.1 & 0.51 & 130 & 200 & {$[44]$} \\
\hline
\end{tabular}

Table II. A compilation of the $k \cdot p$ parameter values reported across spin-orbit coupled topological materials.

Finally, we compare the surface state $k \cdot p$ parameters of $\mathrm{Sb}$ to those obtained from conventional Dirac cone TIs (Tbl. II). The $k \cdot p$ parameters are consistent with the observed LL and QPI phenomenology - the relatively large ratio of $v_{0} / \alpha$ in Sb signifies the presence of an outer surface state Rashba cone which eventually folds over to isolate a linearly dispersing Dirac cone with reduced higher order corrections; and the large $\lambda$ results in intra-cone scattering modes $\left(q_{\Gamma-\mathrm{M}, 2}(\varepsilon)\right.$ and $\left.q_{\Gamma-\mathrm{K}}(\varepsilon)\right)$ being observed closer to the Dirac point than in the TIs.

\section{G. DISCUSSION}

In summary, the topological semimetal Sb hosts pristine topological surface states, screened from chemical potential fluctuations by the bulk states. Moreover, its negative band gap results in the crossover of the surface state band structure from Rashba-like at low energies to Dirac-like at higher energies - providing a conceptual link between conventional Rashba bands and an isolated Dirac cone. Our momentum-resolved STM studies have delved into several idiosyncrasies stemming from the dual Rashba-Dirac nature of Sb surface states - most notably the crossover behavior observed in the LL and QPI dispersions, and the unique provision of momentum-resolved 
STM access to near-Dirac point physics with and without external magnetic fields. This provides a conceptual framework to investigate and engineer tunable Rashba states with topological properties[25, 26].

*anjan@alum.mit.edu

† jhoffman@physics.harvard.edu

[1] M. Z. Hasan and C. L. Kane, Reviews of Modern Physics 82, 3045 (2010).

[2] X.-L. Qi and S.-C. Zhang, Reviews of Modern Physics 83, 1057 (2011).

[3] J. E. Moore, Nature 464, 194 (2010).

[4] Y. Ando, J. Phys. Soc. Japan 82, 102001 (2013).

[5] L. Fu, C. L. Kane, and E. Mele, Physical Review Letters 98, 106803 (2007).

[6] L. Fu and C. L. Kane, Physical Review B 76, 045302 (2007).

[7] E. I. Rashba, Soviet Physics, Solid State 2, 1109 (1960).

[8] L. Fu, Physical Review Letters 103, 266801 (2009).

[9] R. Winkler, Spin-orbit Coupling Effects in TwoDimensional Electron and Hole Systems (Springer, 2003) p. 240.

[10] H. J. Zhang, C.-X. Liu, X.-L. Qi, X. Dai, Z. Fang, and S.-C. Zhang, Nature Physics 5, 438 (2009).

[11] Y. L. Chen, J. G. Analytis, J.-H. Chu, Z. K. Liu, S.-K. Mo, X.-L. Qi, H. J. Zhang, D. H. Lu, X. Dai, Z. Fang, S.-C. Zhang, I. R. Fisher, Z. Hussain, and Z.-X. Shen, Science 325, 178 (2009).

[12] Z. Alpichshev, J. G. Analytis, J.-H. Chu, I. R. Fisher, Y. L. Chen, Z.-X. Shen, A. Fang, and A. Kapitulnik, Physical Review Letters 104, 016401 (2010).

[13] D. Hsieh, Y. Xia, L. A. Wray, D. Qian, A. Pal, J. H. Dil, J. Osterwalder, F. Meier, G. Bihlmayer, C. L. Kane, Y. S. Hor, R. J. Cava, and M. Z. Hasan, Science 323, 919 (2009).

[14] D. Hsieh, Y. Xia, D. Qian, L. A. Wray, J. H. Dil, F. Meier, J. Osterwalder, L. Patthey, J. G. Checkelsky, N. P. Ong, A. V. Fedorov, H. Lin, A. Bansil, D. Grauer, Y. S. Hor, R. J. Cava, and M. Z. Hasan, Nature 460, 1101 (2009).

[15] Y. Jiang, Y. Wang, M. Chen, Z. Li, C. Song, K. He, L.-L. Wang, X. Chen, X.-C. Ma, and Q.-K. Xue, Physical Review Letters 108, 016401 (2012).

[16] J. G. Checkelsky, Y. S. Hor, M.-H. Liu, D.-X. Qu, R. J. Cava, and N. P. Ong, Physical Review Letters 103, 246601 (2009).

[17] H. Beidenkopf, P. Roushan, J. Seo, L. Gorman, I. Drozdov, Y. S. Hor, R. J. Cava, and A. Yazdani, Nature Physics 7, 939 (2011).

[18] S. Jia, H. Beidenkopf, I. Drozdov, M. Fuccillo, J. Seo, J. Xiong, N. Ong, A. Yazdani, and R. Cava, Physical Review B 86, 165119 (2012).

[19] T. Arakane, T. Sato, S. Souma, K. Kosaka, K. Nakayama, M. Komatsu, T. Takahashi, Z. Ren, K. Segawa, and Y. Ando, Nature Communications
3, $636(2012)$

[20] Z.-H. Zhu, G. Levy, B. Ludbrook, C. Veenstra, J. Rosen, R. Comin, D. Wong, P. Dosanjh, A. Ubaldini, P. Syers, N. P. Butch, J. Paglione, I. Elfimov, and A. Damascelli, Physical Review Letters 107, 1 (2011).

[21] M. Bianchi, D. Guan, S. Bao, J. Mi, B. B. Iversen, P. D. C. King, and P. Hofmann, Nature Communications 1, 128 (2010).

[22] K. Ishizaka, M. S. Bahramy, H. Murakawa, M. Sakano, T. Shimojima, T. Sonobe, K. Koizumi, S. Shin, H. Miyahara, A. Kimura, K. Miyamoto, T. Okuda, H. Namatame, M. Taniguchi, R. Arita, N. Nagaosa, K. Kobayashi, Y. Murakami, R. Kumai, Y. Kaneko, Y. Onose, and Y. Tokura, Nature Materials 10, 521 (2011).

[23] H. Murakawa, M. S. Bahramy, M. Tokunaga, Y. Kohama, C. Bell, Y. Kaneko, N. Nagaosa, H. Y. Hwang, and Y. Tokura, Science 342, 1490 (2013).

[24] Y. L. Chen, M. Kanou, Z. K. Liu, H. J. Zhang, J. A. Sobota, D. Leuenberger, S. K. Mo, B. Zhou, S.-L. Yang, P. S. Kirchmann, D. H. Lu, R. G. Moore, Z. Hussain, Z. X. Shen, X. L. Qi, and T. Sasagawa, Nature Physics 9, 704 (2013).

[25] T. Das and A. V. Balatsky, Nature Communications 4, 1972 (2013).

[26] B. Rasche, A. Isaeva, M. Ruck, S. Borisenko, V. Zabolotnyy, B. Büchner, K. Koepernik, C. Ortix, M. Richter, and J. van den Brink, Nature Materials 12, 422 (2013).

[27] Z. F. Wang, M.-Y. Yao, W. Ming, L. Miao, F. Zhu, C. Liu, C. L. Gao, D. Qian, J.-F. Jia, and F. Liu, Nature Communications 4, 1384 (2013).

[28] D. Hsieh, L. A. Wray, D. Qian, Y. Xia, J. H. Dil, F. Meier, L. Patthey, J. Osterwalder, G. Bihlmayer, Y. S. Hor, R. J. Cava, and M. Z. Hasan, New Journal of Physics 12, 125001 (2010).

[29] C. J. Chen, Introduction to Scanning Tunneling Microscopy (Monographs on the Physics and Chemistry of Materials), 2nd ed. (Oxford University Press, 2007).

[30] J. Bardeen, Physical Review Letters 6, 57 (1961).

[31] M. Morgenstern, C. Wittneven, R. Dombrowski, and R. Wiesendanger, Physical Review Letters 84, 5588 (2000)

[32] T. Hanaguri, K. Igarashi, M. Kawamura, H. Takagi, and T. Sasagawa, Physical Review B 82, 081305 (2010).

[33] D. L. Miller, K. D. Kubista, G. M. Rutter, M. Ruan, W. A. de Heer, P. N. First, and J. A. Stroscio, Science 324, 924 (2009).

[34] Y. Okada, W. Zhou, C. Dhital, D. Walkup, Y. Ran, Z. Wang, S. D. Wilson, and V. Madhavan, Physical Review Letters 109, 166407 (2012).

[35] M. F. Crommie, C. P. Lutz, and D. M. Eigler, Nature 363, 524 (1993).

[36] J. E. Hoffman, K. McElroy, D.-H. Lee, K. M. Lang, H. Eisaki, S. Uchida, and J. C. Davis, Science 297, 1148 (2002).

[37] K. McElroy, G.-H. Gweon, S. Zhou, J. Graf, S. Uchida, H. Eisaki, H. Takagi, T. Sasagawa, D.H. Lee, and A. Lanzara, Physical Review Letters 
96, 067005 (2006).

[38] G. M. Rutter, J. N. Crain, N. P. Guisinger, T. Li, P. N. First, and J. A. Stroscio, Science 317, 219 (2007).

[39] P. Roushan, J. Seo, C. V. Parker, Y. S. Hor, D. Hsieh, D. Qian, A. Richardella, M. Z. Hasan, R. J. Cava, and A. Yazdani, Nature 460, 1106 (2009).

[40] T. Zhang, P. Cheng, X. Chen, J.-F. Jia, X.-C. Ma, K. He, L.-L. Wang, H.-J. Zhang, X. Dai, Z. Fang, X.-C. Xie, and Q.-K. Xue, Physical Review Letters 103, 266803 (2009).

[41] Y. Okada, C. Dhital, W. Zhou, E. D. Huemiller, H. Lin, S. Basak, A. Bansil, Y.-B. Huang, H. Ding, Z. Wang, S. D. Wilson, and V. Madhavan, Physical Review Letters 106, 206805 (2011).

[42] Y. Zhang, V. W. Brar, C. O. Girit, A. Zettl, and M. F. Crommie, Nature Physics 5, 722 (2009).

[43] Y. Okada, W. Zhou, D. Walkup, C. Dhital, S. D. Wilson, and V. Madhavan, Nature Communications 3, 1158 (2012).

[44] A. Soumyanarayanan, M. M. Yee, Y. He, H. Lin, D. R. Gardner, A. Bansil, Y. S. Lee, and J. E. Hoffman, ArXiv e-prints (2013), arXiv:1311.1758 [condmat.mes-hall].

[45] STM measurements were performed using a homebuilt instrument at liquid helium temperatures using Pt-Ir tips. Single crystals of Sb were grown using the modified Bridgman method[13], and cleaved in-situ in cryogenic ultrahigh-vacuum to expose the (111) face[28]. The majority of the results on Sb shown herein have been presented previously[44].

[46] P. Cheng, C. Song, T. Zhang, Y. Zhang, Y. Wang, J.-F. Jia, J. Wang, Y. Wang, B.-F. Zhu, X. Chen, X.C. Ma, K. He, L.-L. Wang, X. Dai, Z. Fang, X. Xie, X.-L. Qi, C.-X. Liu, S.-C. Zhang, and Q.-K. Xue, Physical Review Letters 105, 076801 (2010).

[47] Y.-S. Fu, T. Hanaguri, S. Yamamoto, K. Igarashi, H. Takagi, and T. Sasagawa, ACS Nano 7, 4105 (2013).
[48] C.-X. Liu, X.-L. Qi, H. Zhang, X. Dai, Z. Fang, and S.-C. Zhang, Physical Review B 82, 045122 (2010).

[49] J. Schliemann, J. C. Egues, and D. Loss, Physical Review B 67, 085302 (2003).

[50] M. Zarea and S. E. Ulloa, Physical Review B 72, 085342 (2005).

[51] M. Morgenstern, A. Georgi, C. Straß er, C. Ast, S. Becker, and M. Liebmann, Physica E 44, 1795 (2012).

[52] K. K. Gomes, W. Ko, W. Mar, Y. L. Chen, Z.-X. Shen, and H. C. Manoharan, ArXiv e-prints (2009), arXiv:0909.0921 [cond-mat.mes-hall].

[53] J. Seo, P. Roushan, H. Beidenkopf, Y. S. Hor, R. J. Cava, and A. Yazdani, Nature 466, 343 (2010).

[54] G. Yao, Z. Luo, F. Pan, W. Xu, Y. P. Feng, and X.-S. Wang, Scientific Reports 3, 02010 (2013).

[55] A. Narayan, I. Rungger, and S. Sanvito, Physical Review B 86, 201402 (2012).

[56] L. El-Kareh, P. Sessi, T. Bathon, and M. Bode, Physical Review Letters 110, 176803 (2013).

[57] P. D. C. King, R. Hatch, M. Bianchi, R. Ovsyannikov, C. Lupulescu, G. Landolt, B. Slomski, J. Dil, D. Guan, J. Mi, E. Rienks, J. Fink, A. Lindblad, S. Svensson, S. Bao, G. Balakrishnan, B. Iversen, J. Osterwalder, W. Eberhardt, F. Baumberger, and P. Hofmann, Physical Review Letters 107, 096802 (2011).

[58] Y. Wang, D. Hsieh, D. Pilon, L. Fu, D. R. Gardner, Y. S. Lee, and N. Gedik, Physical Review Letters 107, 207602 (2011).

Acknowledgements. We are grateful to collaborators Michael Yee and Yang He for their contributions to the STM experiments[44]; and Hsin Lin and Jason Zhu for theoretical inputs and useful discussions. The STM work at Harvard was supported by the NSF under Grant No. DMR-1106023, and A.S. acknowledges funding from A*STAR, Singapore. 